\title{
Blockchain Technology in Education System: A Review
}

\author{
Akshay Karale \\ Department of Computer Engineering, Marathwada \\ Mitra Mandal's College of Engineering \\ Pune-400041
}

\author{
Harmeet Khanuja \\ Department of Computer Engineering, Marathwada \\ Mitra Mandal's College of Engineering \\ Pune-400041
}

\begin{abstract}
Blockchain technology can be used to solve many educational problems and can help educators as well as learners to monitor the learning outcomes. The data can be stored securely and tamper proof format when it's stored onto the blockchain network. The Blockchain for Education platform helps us to make the tamper-proof certificates and their correct and the overall permanent allocation of these certificates to learners, as well as verification of certificates. It can reduce the overall frauds and tampering of the degrees and certificates. Here smart contracts can be designed and deployed on to the Ethereum blockchain that can be designed using the solidity programming language. Blockchain can be applied to private, public and consortium sectors depending upon the usage and the scope of the blockchain. Education system can take benefit of this scalability of the blockchain and can be effectively useful in the
\end{abstract}

\section{Keywords}

Blockchain, Ethereum, Distributed ledgers, Smart contracts, Solidity, POW, Consensus.

\section{INTRODUCTION}

We ask Blockchain can transform the traditional record storage of students and the staff over the distributed network. As the data is stored on the distributed system it is secure and more transparent. The overall data accuracy and immutability is preserved in the distributed blockchain environment. Generally blockchain consist of the various steps while deploying the data onto the blockchain network. Here each user has their own hash value associated which will help them to identify them uniquely over the distributed network. The time stamp is also added as the part of the hash value which is generally from random value. Issues in the current education system and different educational organizations can be resolved using the blockchain technology in the core area. It can be resolved using the blockchain technology in education system. Usually the verifying authorities and the central institutions take more amount of time for carrying out the operations which is a time consuming process. Using the blockchain technology we can eliminate the risk of the central server down time. As we are not relying on the central authority the process takes fewer amount of time. The data stored on the blockchain is tamper proof and cannot be modified once deployed on blockchain network.

\section{LITERATURE SURVEY}

The paper [1] depicts the potential application of the blockchain in the education system and organizations. Further explains the different issues in the current education system and different educational organizations can be resolved using the blockchain technology in the core area and how It can be resolved using the blockchain technology in education system. Usually the verifying authorities and the central institutions take more amount of time for carrying out the operations which is a time consuming process. It continues with the blockchain technology we can eliminate the risk of the central server down time. As we are not relying on the central authority the process takes fewer amount of time. Considering that the data stored on the blockchain is tamper proof and cannot be modified once deployed on blockchain network and stored in the form of tamper proof smart contracts. The paper [2] provides the different approaches and ways to consider the consensus as adding the POW (Proof of Work) to the blockchain. Using this algorithm different parties reach to the consensus like to add the corresponding the transaction to the corresponding blockchain or not. Here the difficulty can be increased to solve the cryptographic puzzle and it can be made more challenging to solve by increasing the more number of leading zero's in the overall cryptographic puzzle.

Paper [4] describes how the peer-to-peer electronic cash can help users to send the money from one point to other point without involvement of the third parties. And also added the use of the Ethereum blockchain in the corresponding blockchain network. Further explains how the digital signature and digital certifications can help the educational institutions and disrupt the current centralized systems. Blockchain technology provides more security to the data compared to the central data storage, as we don't need to worry about server down time. Even if we want to hack the overall blockchain network we need to gain the access the $51 \%$ access of the network and which is quite impossible to perform. And blockchain is secured by many number of the active nodes in the blockchain network.

[5] Provides the information about the string of the knowledge is used that cryptographic token. Which generally refers to the creation and the transfer of the cryptocurrencies as well as the storage. Generally the cryptographic token refers to the string of the information which actually points to the knowledge having the initial data. [5] Ether is the fuel in the distributed platform based applications. It is used in the Ethereum blockchain. It further explains that it can be considered as the payment method generally preferred in the Ethereum blockchain network. As it is applied so that developers can develop the quality of the applications. [6]Provides the information regarding the Ethereum blockchain and how it can be implemented in the form of the smart contracts designed using the solidity programming language. [6]Explains the additional and innovative approaches that can made possible using the quality attributes of blockchain technology for the educational systems.

\section{BLOCKCHAIN TERMINOLOGIES}

\subsection{Transactions}

Blockchain contains the shared and the distributed transactional database. When changes are made to data base we have to make a transactions and it has to be agreed and accepted by everyone in the network. The transactions on the network are always cryptographically signed and maintained uniquely. 


\subsection{Distributed Ledgers}

It is a database held and then updated by each and every participant in the comparatively large network. The overall distribution is kind of unique as the communication occurs in distributed way and it's not carried out by the central authority. Here once it is updated all the nodes in the network receives the updated copy of the corresponding ledger.

\subsection{Cryptographic Token}

The string of the knowledge is used that is called as the cryptographic token. Which generally refers to the creation and the transfer of the cryptocurrencies as well as the storage. Generally the cryptographic token refers to the string of the information which actually points to the knowledge having the initial data. It is actually associated with all the blocks in corresponding blockchain.[6]

\subsection{Ether}

Ether is the fuel in the distributed platform based applications. It is used in the Ethereum blockchain. It can be considered as the payment method generally preferred in the Ethereum blockchain network. As it is applied so that developers can develop the quality applications and the overall network remains healthy and the transfer performs in the correct manner. [6]

\subsection{Ethereum Blockchain}

Ethereum is platform which follows the decentralized platform and it can be accessed by deploying the smart contracts. Here we have no need to worry about server down time. As the smart contracts are shared by all the nodes in the network. Here we have the different programming languages to design smart contracts which can be efficiently deployed on Ethereum blockchain. [6]

\subsection{Gas}

To limit the overall amount of work done, each transaction is associated with the certain amount of the gas. The value of the gas is calculated with the formula (gas price * gas).The moment if an out of gas notification is triggered then it'll revert all the corresponding transaction at the given moment. Usually the actual price of the gas is set by the one who has created the transaction.

\subsection{Smart Contract}

Smart contracts are the scripts which are self-executing and can be written in programming languages such as the JavaScript, python or solidity. Smart contracts are generally used to specify the rules while to parties communicate with each other. It can overall reduce the degree of security and helps us to lower the transaction cost.

\subsection{Solidity}

Solidity is the object oriented programming language and it has syntax which resembles mostly to the languages like the $\mathrm{c}++$, python and JavaScript. Generally the remix IDE is used to write the smart contracts in the solidity. It has various inbuilt tools which helps the environment more active and useful for handling the solidity programming environment.

\section{BLOCKCHAIN QUALITY ATTRIBUTES 4.1 Increased Capacity}

The blockchain can remarkably increase the overall capacity of the entire network. As it runs on the principal of distributed computing, the overall power of the entire network can be a plus point which can offers us the overall great power and it's more effective than centralized systems.

\subsection{Better Security}

Blockchain technology provides more security to the data compared to the central data storage, as we don't need to worry about server down time. Even if we want to hack the overall blockchain network we need to gain the access the $51 \%$ access of the network and which is quite impossible to perform. And blockchain is secured by many number of the active nodes in the blockchain network.

\subsection{Immutability}

Immutable ledgers is one of the important aspect in the blockchain technology. Usually any database which has a central system is prone to attack. In the blockchain the data is stored as in the distributed ledgers system which will make it more to tamper proof and transparent among all the parties. It has the smart contracts which are self-executing scripts and they will help to make the overall blockchain network more stable and immutable. As all the ledgers are kept up to date and has all the copies shared among all the nodes in the corresponding blockchain network. [6]

\subsection{Faster Settlement}

In the traditional systems usually the settlement cost is more and as its central authority based systems the settlement time is more compared to the distributed networking in the blockchain. Here in the blockchain we do not need to depend on the central authorities and it will overall decrease the settlement cost and time also increase speed and efficiency of transactions.

\subsection{Decentralized System}

Decentralized systems usually provides us many advantages over the traditional centrally managed systems. Blockchain can provide the overall power to the actual owner of the data by giving him unique ID to access the data over the blockchain network. Blockchain technology provide the great functionally with the overall tamper proof data and it will help all the parties to keep the transactions in the network transparent. It can help to bring the massive and remarkable changes in the current industries.

\section{ALGORITHM}

\subsection{Proof of Work (POW): Introduction}

Proof of Work (POW) is the consensus algorithm which is used by many of the popular cryptocurrencies. Using this algorithm different parties reach to the consensus like to add the corresponding the transaction to the corresponding blockchain or not. Here the difficulty can be increased to solve the cryptographic puzzle and it can be made more challenging to solve by increasing the more number of leading zero's in the overall cryptographic puzzle. It usually refers to the overall computing power a specific user has and if the user can solve puzzle in lesser amount of time he will be awarded with the certain amount of cryptocurrencies. More advanced algorithms to proof of work are proof of concept (POC) and proof of statement (POS.

\subsection{Proof of Work: Working}

It is used to confirm transactions and once it is confirmed new blocks are added to the blockchain. Mathematical puzzle requires more amount of computational power and resources. The solution to the proof of work or mathematical equation is called as the 'hash value'. The benefits of using the POW is the anti-dos attacks defense and the low impact of stake for the mining possibilities 
The following are the types of puzzle:

1. Finding hash function.

2. Finding input knowing the corresponding output

3. Presenting a number as the multiplication of the corresponding previous values.

\section{BLOCKCHAIN IN EDUCATION}

In education systems the certificates states that the achievements of the learners or educators and different activities are mostly issued on paper or other physical formats. Paper certificates are prone to tampering and forging. The digital certificates stored onto the blockchain networks are difficult to forge and they are tamper-proof [2]. Here we do not need the third parties to verify the details. Here we don't have central data base to store all the data or to maintain the registries for longer period of time.

Blockchain technology supports protection against counterfeit certificates and simplified verification of Certificates with lesser amount of the resources. Blockchain consist of three steps while considering the digital certification and storing the data onto the blockchain. First we need to identify the certification. Then these certification authorities have to issue certificates to educators and learners.

And lastly the main task is the verification of certificates by educators and learners. Mostly the learners receive the paper certificates with built-in security features, so maintaining the integrity and the immutability of the data can be challenging task. It can be resolved using the blockchain technology in education system.

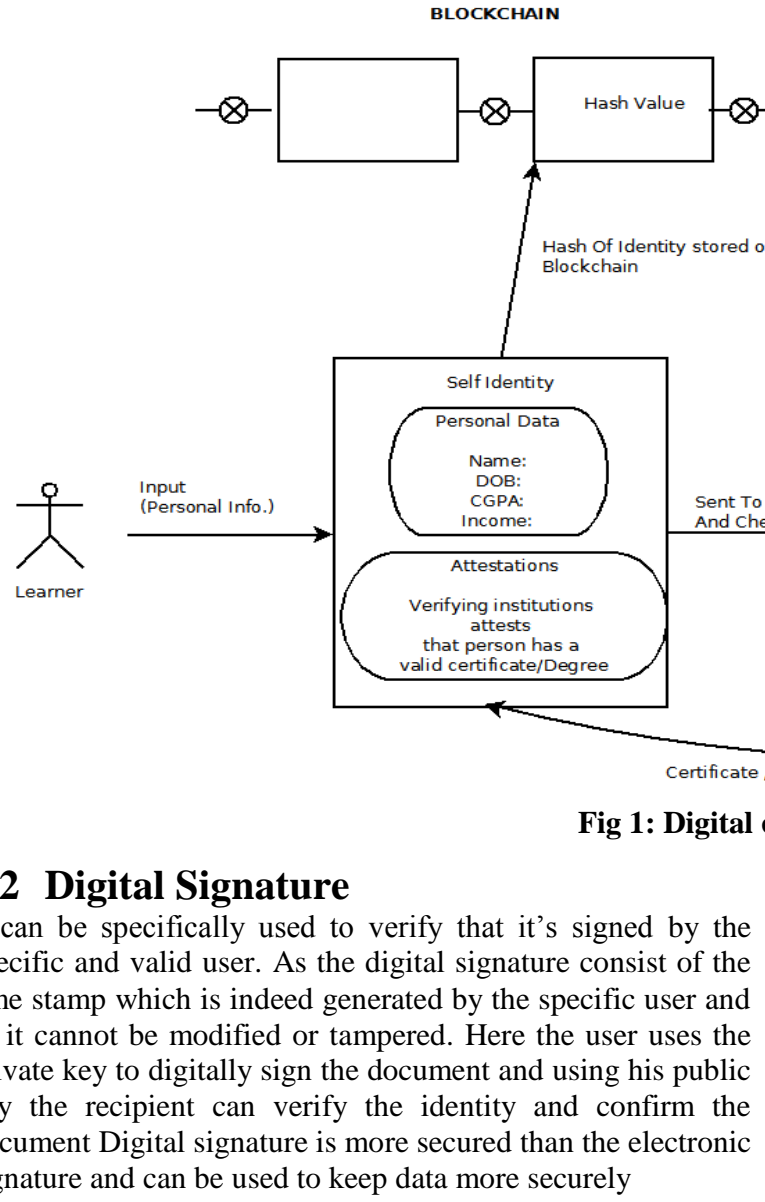

Educators only receives the copies or notarized work of learner's paper certificates and to verify these certificates the educators need to rely on the central authority and it is time consuming process, so the blockchain technology can be effectively used in education system. Currently the Sony and University of Nicosia has successfully implemented the blockchain in education for storing the digital certificate and issuing the degrees. [1]

\section{IMPLEMENTATION OF BLOCKCHAIN IN EDUCATION}

\subsection{Digital Certification}

\subsubsection{Digital Certification Working}

Initially the learner inputs his personal information with the unique id and then the information is kept on to the learner's Blockchain. Later the data sent for the verification to the respective verifying institution. After successful verification of the data the success token is sent to the learner and then the data is safely stored onto the institute's private blockchain network.

\subsubsection{Important Characteristics of Digital Certificates}

The certificates are stored digitally onto the blockchain network with the generated hash. Here the issuer and the verifying authority has their own separate blockchain for storing the digital certificates. Over the regular certificates they are stored digitally on to the thousands of the computers which will make it more secured and powerful than the normal certificates. It requires fewer resources to maintain, issue and access than the existing digital certification.

BLocKCHAIN

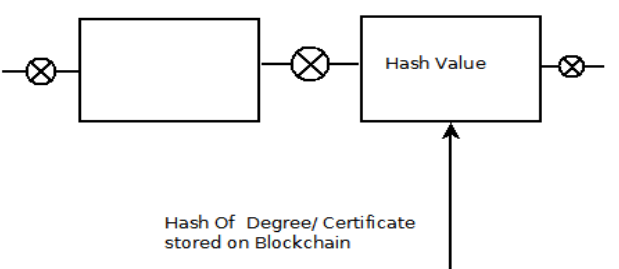




\subsection{Distributed Database in Educational Environment}

Blockchain ledger is distributed so any changes made to them can be monitored by the remaining candidates, so it can reduce the problems like degree frauds. As the overall data is stored on distributed blockchain network, all the learners can keep the track of the details so the overall tampering of the actual data can be avoided, as the blocks on the blockchain cannot be altered once they are deployed. Using the proof of work algorithm the data stored on the distributed blockchain network can be verified and then it'll be stored on the network with valid credentials.

\section{SYSTEM WORKING OVERVIEW}

\subsection{Learners' Module}

The learner consist of their own ID which will help them to uniquely identify them on blockchain network. They are provided with the different rights while storing the data on to blockchain. Learners can inert the theory attendance, insert assignment marks, insert lab and test attendances. After confirming the identity of the learner through the verifying institution he can successfully store the data onto the blockchain using the smart contracts.

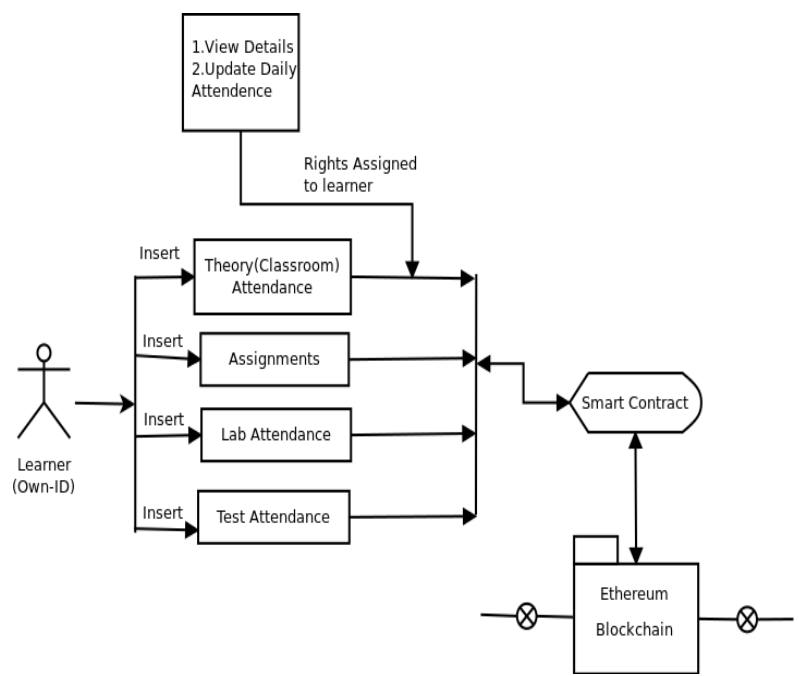

Fig. 2 Learner's module on blockchain

\section{FUTURE EDUCATIONAL APPLICATIONS}

- "Learning is earning" can be applied by considering the digital currencies in the learner's wallet and it can motivate learners to join different educational activities.

- Formative assessment is kind of challenging as we need to record all the details of the learner's activities. It can be made more simplified and can be used in more systematic way in educational system.

- We can maintain the digital identity of the different these parties safe and it can be securely stored on to the blockchain network.

- The smart contract will reduce the overall need of the involvement of the third-party and increases the overall performances.

- The more number of the educational issues can be solved using the smart contract based blockchain systems.

- Every detail of teaching and learning can be made more simplified using the blockchain techniques.

\section{CONCLUSIONS}

Blockchain works on distributed technology. In that different cryptographic techniques and different consensus algorithm such as proof of work (POF) is used. Blockchain provides decentralization, tamper-proof data storage, easily scalability of the data and also provides immutability so it can be effectively used for education system. Blockchain can be used for storing the certificates and degrees. It can reduce the overall frauds and tampering of the degrees and certificates. Here smart contracts can be designed and deployed on to the Ethereum blockchain that can be designed using the solidity programming language. Blockchain can be applied to private, public and consortium sectors depending upon the usage and the scope of the blockchain. Education system can take benefit of this scalability of the blockchain and can be effectively useful in the educational institutions.

\section{REFERENCES}

[1] Guang Cheng, Bing Xu, Manli Lu and Nian Shing Chen, 2018 , Exploring Blockchain technology and its potential applications for education Springer.

[2] Rishav Chatterjee, Rajdeep Chatterjee, 2017, Overview of the emerging Technology: Blockchain .IEEE.

[3] Alexander Grech, Anthony F. Camilleri, 2017 Blockchain in Education.

[4] Satoshi Nakamoto, 2008, Bitcoin: A Peer-to-Peer Electronic Cash System, White paper.

[5] F. Tschorsch and B. Scheuermann, 2016 Bitcoin and beyond: a Technical Survey on decentralized digital currencies, IEEE.

[6] Data Flair, 2018 Web link: https://data flair.training/blogs/types-of- blockchain.

[7] Allen Ezell and John Bear, 2018 .Blockchain for Education: Lifelong Learning Passport - dot magazine.

[8] D. Eastlake, 3rd and T. Hansen, US Secure Hash Algorithms and SHA (Informational).

[9] G. Wood, Ethereum: 2018, A secure decentralized generalized transaction Ledger used in blockchain, Byzantium version.

[10] Agility A. 2017, Proof-of-Knowledge: same Blockchain, different .Available at:https://tail.aqquadro.it/proof-ofknowledge 